\title{
CIELAB based Tone-Mapping Algorithm for LDR Image Generation
}

\author{
Manoj Kumar Patle \\ Scope College of Engineering, Bhopal, India
}

\author{
Anshuj Jain \\ Scope college of Engineering, Bhopal, India
}

\begin{abstract}
This paper presents a analysis of the CIELAB color feature based tone mapping technique. The basic tone mapping was generally the reverse going to the old technology objects compatibility. The compatibility is the basic requirement of the market to grow with the latest technology and the old objects to work with new technology. The paper worked for maintaining this compatibility. The basic theme of the work is done in the SCIELAB color space. The proposed method has done the tone mapping through the decomposition of the image in this SCIELAB color space. The Visual quality of the proposed method is same as of saliency based tone mapping but it is more computational efficient.
\end{abstract}

\section{Keywords}

Tone mapping, Image color space, LDR generation, HDR image processing

\section{INTRODUCTION}

There are various high quality images with high dynamic range available. The display devices problem may come as in the conventional devices. To solve this problem the tonemapping is the approach. This approach provides the good compatibility with maintaining the quality of image. Wei et al. [1] presents a two layer local inverse Tone Mapping assessment algorithm by decomposing via edge-preserving method. $\mathrm{Ke} \mathrm{Gu}$ et al. [2] provides a High dynamic range (HDR) imaging methods working actively. They developed an objective quality metric based LDR images creation by many TMOs without accessing original HDR images. Ma et al. [3] developed a tone mapping operators (TMOs) for high dynamic range (HDR) to low dynamic range (LDR) images. Here it is designed a TMO that navigate in the space domain for all images, and optimizes an improved TMQI. It then uses an iterative process that improves the structural fidelity alternatively of the image.

Nafchi [4] worked on feature similarity index for tone-mapped images (FSITM), to estimate a tone mapping operator (TMO). The index matches the high dynamic range (HDR) image map based on the locally weighted mean phase angle. That may be complex in the processing. Li et al. [5] developed a novel saliency-aware weighting with weight of edges are introduced for HDR images. This method mainly works on the Halo artifact that is reduced in the tone-mapped image. Wang et al. [6] proposed a region-based improvement on the pseudoexposures to enhance niceties in the distinct region. The fusion [11] is done by region-enriched pseudo-exposures into an HDR image. Boschetti et al. [7] worked on the tone map procedure, derived through Contrast Limited Adaptive Histogram Equalization (CLAHE) method. The comparison of obtained LDR images, with those obtained by State of the Art TMOs, has the ability to equally enhance visibility in both dark and bright areas. Huang et al. [8] evolved a tone mapping technique which combines multi-scale base-detail decomposition and bilateral grid filtering. Jinno et al. [9] work on the HDRI image acquisition. They used the multiple exposure fusion [11] technique to boost the dynamic range of image. The high dynamic range images (HDRIs) construction is achieved by combining many images opted with different exposures and assessing the irradiance parameter from each pixel value. An adaptive local tone mapping method that selects the vicinity of current pixel adaptively to reduce artifacts near edges is used for tone mappping [10-14]. The local parameter based method helps in the quality wise tone mapping enhancement. B. Gu et al. [15] proposed a filter with preserved edge image decomposition. It has its locally adaptive belongings. The algorithm is preserving effective local details and enhancing quality. Yeganeh et al. [16] worked on the tone-mapping operators (TMOs) that are used to convert the high dynamic range (HDR) to low dynamic range (LDR) images and provide beneficial tactics for visualization of HDR images LDR displays. This is combining the multiscale signal fidelity of a modified structural similarity index and naturalness measure of intensity statistics in natural images.

The tone mapping operators (TMOs) [17] that searches in the images data set to catch better quality images in terms of a recent objective degree that can evaluate the structural fidelity in two images of different dynamic ranges. The method uses the gradient ascent direction for searching up to convergence for maximal point. Chakrabarti et al. [18] introduced a model for the uncertainty and a technique for fitting for imaging pipeline. As the model fits, it is gives pixel based tonemapped digital photograph. It introduced a probability distribution of linear scene. These distributions are important for visual inference of vision tasks.

Xiao et al. [19] proposed a colour appearance based hierarchical tone mapping system. The bilateral filter is speed up by Gaussian kernel in discrete form. The tone mapping in colour space of RGB is adapted to precise the colour casts.

$\mathrm{Wu}$ et al. [20] proposed a real time system to form 8-bit images from 12-bit image that preserves both the ample details and high contrast of image. Agrawal et al. [21] propose two operators which are based on different local contrast measures. The operators can compress the HD range, preserving the details high and low intensity regions of image. They compare the HDR and LDR contrast differences by using an online metric [22-26].

The remainder of this paper is organized as follows. In the next section we discuss the background and various applications of base-detail decompositions. Next, in Section 3 we show HDR tone mapping. Section 4 presents a detailed result comparison between our WLS-based multi-scale decompositions with previous scheme, while Section 5 discusses utility of method and conclusion. 


\section{BACKGROUND OF METHOD}

In computational photography, images are often decomposed into a piecewise smooth base layer and one or more detail layers. The base layer captures the larger scale variations in intensity, and is typically computed by applying an edgepreserving smoothing operator to the image (sometimes applied to the logarithm of the luminance or to the lightness channel of the CIELAB color space). Furthermore, in order to produce multi-scale base-detail decompositions, the operator must allow increasingly larger image features to migrate from the base layer to the detail layer. In other words, it must allow increasingly larger regions to become increasingly smoother.

Using more levels would enable finer control, but would also require the user to manipulate more sliders.

\subsection{Edge-Preserving Smoothing via WLS}

In this section, we first describe an alternative edge-preserving smoothing approach based on the WLS optimization framework. Formally, this may be expressed as seeking the minimum of

$\sum_{p}\left(u_{p}-g_{p}\right)^{2}+\left(a_{x, p}(g)\left(\frac{u}{x}\right)^{2}+a_{y, p}(g)\left(\frac{u}{y}\right)^{2}\right)$

where the subscript $\mathrm{p}$ denotes the spatial location of a pixel. The goal of the data term $\left(u_{p}-g_{p}\right)^{2}$ is to minimize the distance between $\mathrm{u}$ and $\mathrm{g}$, while the second (regularization) term strives to achieve smoothness by minimizing the partial derivatives of $u$. Finally, is responsible for the balance between the two terms; increasing the value of 1 results in progressively smoother images $u$.

$$
\begin{gathered}
a_{x, p}(g)=\left(\left|\frac{l}{x}(p)\right|^{\alpha}+\varepsilon\right)^{-1} a_{y, p}(g)= \\
\left(\left|\frac{l}{y}(p)\right|^{\alpha}+\varepsilon\right)^{-1}
\end{gathered}
$$

where $a$ is the log-luminance channel of the input image g, the exponent a (typically between 1.2 and 2.0) determines the sensitivity to the gradients of $\mathrm{g}$, while e is a small constant (typically 0.0001 ) that prevents division by zero in areas where $\mathrm{g}$ is constant.

\subsection{Multi-scale Dcompositions}

Using the edge-preserving operator described above more specifically, let $g$ denote the input image for which we would like to construct a (k+1)-level decomposition. Let $u^{1} ; \ldots ; u^{k}$ denote progressively coarser versions of $g$. The coarsest of these versions, $u^{k}$ will serve as the base layer $\mathrm{b}$, with the $\mathrm{k}$ detail layers defined as $d^{1}=u^{i-1}-u^{i}$ where $i=1, \ldots, k$ and $u^{0}=g$. the original image $\mathrm{g}$ is easily recovered from this decomposition by simply adding up the base and the detail layers:

$$
g=b+\sum_{i=1}^{k} d^{i}
$$

Note that we do not perform any downsampling of the smoothed images $u_{i}$, since they are obtained via edgepreserving smoothing and are not band-limited in the usual sense. Thus, our multi-scale decomposition is an overcomplete description of the input image.

\subsection{Multi-Scale Tone Manipulation}

Here we first construct a three-level decomposition (coarse base level $b$ and two detail levels $d 1 ; d 2$ ) of the CIELAB lightness channel. The result of the manipulation $\hat{g}$ at each pixel $\mathrm{p}$ is then given by

$$
\hat{g}_{p}=\uparrow \mu+S\left(\delta_{0} b_{p}-\mu\right)+S\left(\delta_{1} d_{p}^{1}\right)+S\left(\delta_{2} d_{p}^{2}\right)
$$

Where $\mathrm{m}$ is the mean of the lightness range, and $\mathrm{S}$ is a sigmoid curve, $\quad S(a ; x)=1=(1+\exp (-a x))$ (appropriately shifted and normalized). The goal of this sigmoid is to avoid the hard clipping that would otherwise occur when the detail layers are significantly boosted. The term $\mathrm{S}(\delta ; \eta \eta \mathrm{bp}-\mu)$ controls the exposure and contrast of the base layer, while the remaining terms control the boosting of the medium and fine scale details. Using more levels would enable finer control, but would also require the user to manipulate more sliders.

\section{HDR TONE MAPPING}

Our decompositions are easily harnessed to perform detail preserving compression of HDR images. For example, we can simply replace the BLF in the tone mapping algorithm of Durand and Dorsey, with WLS-based smoothing and avoid the mild halo artifacts that are sometimes visible in their results.

HDR image as input

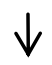

Edge-preservation and decomposition

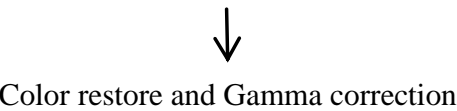<smiles>C[AlH]</smiles>

Filtering and tone mapping<smiles>C[AlH]</smiles>

Output as LDR Image

Fig.1 The Block Diagram of the Proposed Algorithm.

We compute a 4-level decomposition (one coarse base layer and three detail layers) of the log-luminance channel, multiply each level by some scaling factor, and reconstruct a new logluminance channel. We achieved this result by using less compression of the base and moderate boosting of the coarser detail layers.

\subsection{Progressive image abstraction}

Our WLS-based operators use the BLF for image and video abstraction.

$$
u^{i+1}=F_{c^{i}}\left(u^{i}\right)
$$

$F$ a nonlinear operator, which depends on $\mathrm{g}$ is a gradient : for some initial value of and some factor c. A coarsening sequence generated in this manner and the corresponding detail layers. Using our multi-scale decomposition (the iterative version of eq. (1)) produces the results. In this application, the detail layers are attenuated, rather than boosted, to achieve a stylized abstract look. The images are 
overlaid with edges extracted from the appropriate detail layers.

\section{RESULTS}

We have implemented a number of simple tools that use our multiscale edge-preserving decompositions for photographic tone manipulation, HDR tone mapping, detail enhancement, and image abstraction. Below, it is briefly define these apparatuses and show some outcomes. It is noted that the purpose of these apparatuses are to demonstrate in the easiest possible mode for the robustness and versatility of our decompositions.

\subsection{Performance Parameters}

The analysis can be done on the basis of these performance parameters. It is straightforward to estimate the noise variance by the maximum likelihood (ML) criterion. It is given by

$$
\begin{aligned}
& \sigma^{2}=\operatorname{argmax} P\left(Y \mid H, X, \sigma^{2}\right) \\
& =\frac{1}{M N} \sum_{i}\left(Y_{i-} H_{i} X\right)^{T}\left(Y_{i-} H_{i} X\right) .
\end{aligned}
$$

Universal Image Quality Index (UIQI): A UIQI [25] has been widely used for image similarity evaluation and was also applied to validate fusion techniques [26]. UIQI of two images $(A$ and $B)$ is defined as

$$
Q=\frac{4 \sigma_{A B} \mu_{A} \mu_{B}}{\left(\sigma_{A}^{2}+\sigma_{B}^{2}\right)\left(\mu_{A}^{2}+\mu_{B}^{2}\right)}
$$

This quality index models any distortion as a combination of three different factors: loss of correlation, luminance distortion, and contrast distortion. The dynamic range of $Q$ is $[-1,1]$, and the best value 1 is obtained if $A=B$. When applying this index to a multiband image, it is applied bandby-band and averaged over all bands. [7].

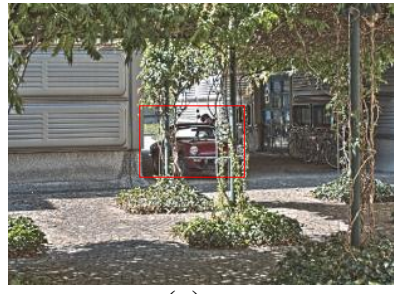

(a)

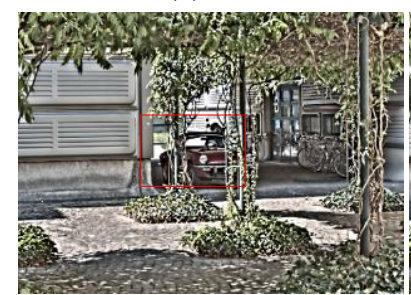

(c)

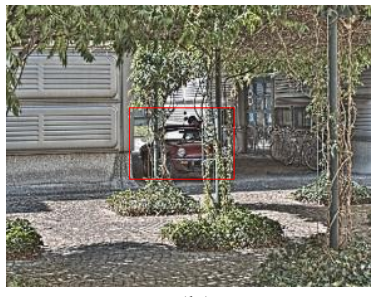

(b)

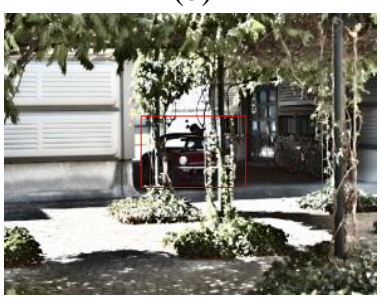

(d)
Fig.2 (a) Result image of the Saliency based tone mapping and (b), (c) and (d) Result image of the propose tone mapping algorithm with fine, medium and coarse detail respectively
Table 1 Performance parameter for car image

\begin{tabular}{|c|c|c|c|}
\hline Method & Mean & Variance & UIQI \\
\hline Saliency TM & 0.4413 & 0.0774 & 0.7090 \\
\hline Proposed & 0.4464 & 0.0586 & 0.9968 \\
\hline
\end{tabular}

Table 2 Performance parameter for office image

\begin{tabular}{|c|c|c|c|}
\hline Method & Mean & Variance & UIQI \\
\hline Saliency TM & 0.5241 & 0.1708 & 0.5651 \\
\hline Proposed & 0.4890 & 0.0807 & 0.8554 \\
\hline
\end{tabular}

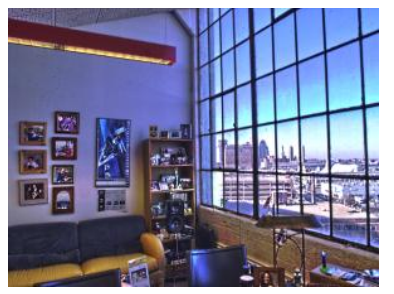

(a)

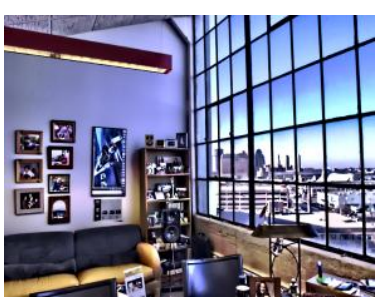

(c)

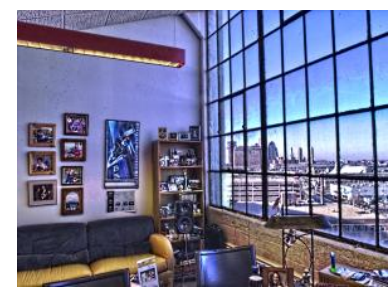

(b)

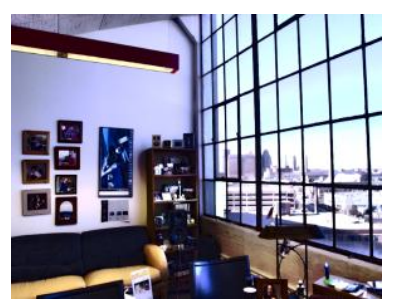

(d)

Fig.3 (a) Result image of the Saliency based tone mapping and (b), (c) and (d) Result image of the propose tone mapping algorithm with fine, medium and coarse detail respectively

\section{CONCLUSION}

The basic theme of the work is done in the SCIELAB color space. The proposed method has done the tone mapping through the decomposition of the image in this SCIELAB color space. The Visual quality of the proposed method is same as of saliency based tone mapping but it is more computational efficient.

\section{REFERENCES}

[1] Z. Wei, C. Wen, and Z. Li, "Local inverse tone mapping for scalable high dynamic range image coding," IEEE Trans. on Circuits and Systems for Video Tech. 2016, pp. 1-7, DOI 10.1109/TCSVT.2016.2611944

[2] K. Gu, S. Wang, G. Zhai, S. Ma, X. Yang, W. Lin, W. Zhang, and W. Gao, "Blind quality assessment of tonemapped images via analysis of information, naturalness, and structure," IEEE Trans. on Mult., vol. 18, no. 3, Mar. 2016, pp. 432-443.

[3] K. Ma, H. Yeganeh, K. Zeng, and Z. Wang, "High dynamic range image compression by optimizing tone mapped image quality index," IEEE Trans. on Image Process., vol. 24, no. 10, Oct. 2015, pp. 3086-3097.

[4] H. Z. Nafchi, A. Shahkolaei, R. F. Moghaddam, and M. Cheriet, "FSITM: A feature similarity index for tonemapped images," IEEE Signal Process. Let. vol. 22, no. 8, Aug. 2015, pp. 1026-1029.

[5] Z. Li, and J. Zheng, "Visual-salience-based tone mapping for high dynamic range images," IEEE Trans. on 
Industrial Electronics, vol. 61, no. 12, Dec. 2014, pp.7076-7082.

[6] T.-H. Wang, C.-W. Chiu, W.-C. Wu, J.-W. Wang, C.-Y. Lin, C.-T. Chiu, and J.-J. Liou, "Pseudo-multipleexposure-based tone fusion with local region adjustment," IEEE Trans. on Multi., vol. 17, no. 4, Apr. 2015, pp. 470-484.

[7] A. Boschetti, N. Adami, R. Leonardi, and M. Okuda, "High dynamic range image tone mapping based on local histogram equalization," ICME 2010, pp. 1130-1135.

[8] P. Huang, Z. Su, and Z. Li, "Multi-scale bilateral grid for image tone mapping," IEEE, 2011, pp. 3143-3146.

[9] T. Jinno and M. Okuda, "Multiple exposure fusion for high dynamic range image acquisition," IEEE Trans. on Image Process., vol. 21, no. 1, Jan. 2012, pp. 358-365.

[10] R. C. Bilcu, S. Alenius and M. Vehvilainen, "Adaptive local tone mapping of color images," 20th Europ. Signal Process. Conf. (EUSIPCO 2012) Bucharest, Romania, August 27 - 31, 2012, pp. 1563-1567.

[11] Y. Kurmi and V. Chaurasia, "An image fusion approach based on adaptive fuzzy logic model with local level processing," Int. Jour. Of Comp. Appl., Aug. 2015, vol. 124, no.1, pp. 39-42.

[12] D. Sharma, Y. Kurmi, and V. Chaurasia, "Formation of super- resolution image: a review," Int. Jour. of Emerging Tech. and Adv. Engg., Apr. 2014, vol. 4, no. 4, pp. 218-221.

[13] Y. Kurmi and V. Chaurasia, "Performance of haze removal filter for hazy and noisy images," Int. Jour. of Sci. Engg. and Tech., Apr. 2014, vol. 3 no. 4, pp. 437439.

[14] S. Tiwari, K. Chauhan, and Y. Kurmi "Shadow detection and compensation in aerial images using MATLAB," Int. Jour. of Comp. Appl., June 2015, vol. 119, no.20, pp. 59.

[15] B. Gu, W. Li, M. Zhu, and M. Wang, "Local edgepreserving multiscale decomposition for high dynamic range image tone mapping," IEEE Trans. on Image Process., vol. 22, no. 1, Jan. 2013, pp. 70-79.
[16] H. Yeganeh and Z. Wang, "Objective quality assessment of tone-mapped images," IEEE Trans. on Image Process., vol. 22, no. 2, Feb. 2013, pp. 657-667.

[17] H. Yeganeh and Z. Wang, "High dynamic range image tone mapping by maximizing a structural fidelity measure," ICASSP 2013, pp. 1879- 1883.

[18] A. Chakrabarti, Y. Xiong, B. Sun, T. Darrell, D. Scharstein, T. Zickler, and K. Saenko, "Modeling radiometric uncertainty for vision with tone-mapped color images," IEEE Trans. on Pattern Analysis and Machine Intell., vol. 36, no. 11, Nov. 2014, pp. 21852198.

[19] J. Xiao, W. Li, G. Liu, S.-L. Shaw, and Y. Zhang, "Hierarchical tone mapping based on image colour appearance model," IET Comput. Vis., 2014, vol. 8, no. 4, pp. 358-364, doi: 10.1049/iet-cvi.2013.0230

[20] Q. Wu, Z. Zhou, H. Leng, J. Cao, J. Wang, Z. Gong, X. Fan, H. Guo, "A novel real-time method for high dynamic range image tone mapping," 2014 IEEE, 2014, pp. 433-436.

[21] A. Agrawal and S. Raman, "A novel LBP based operator for tone mapping HDR images," IEEE 2014.

[22] T. S. Huang, "Coding of two tone images," IEEE Trans. on Commu., Nov. 1977, vol. Com-25, no. 11, pp. 14061424.

[23] C.-H. Lee, E.-Y. Chung, C.-S. Lee, E.-J. Lee, and Y.-H. $\mathrm{Ha}$, "Tone reproduction technique using neural network in inkjet printers," Third International Conference on Knowledge-Based Intelligent Information Engineering Systems, Adelaide, Australia, 31" Aug-1" Sept 1999, pp. 103-106.

[24] J. Duan and G. Qiu, "Fast tone mapping for high dynamic range images," Proceedings of the 17th International Conference on Pattern Recognition (ICPR'04).

[25] A. lranli and M. Pedram, "DTM: Dynamic tone mapping for backlight scaling," Anaheim, California, USA, DAC June 13-17, 2005, pp. 612-617.

[26] M. K. Patle, B. Chourasia, and Y. Kurmi, "High Dynamic Range Image Analysis through Various Tone Mapping Techniques," Int. Jour. of Comp. Appl., vol.153, no. 11 , Nov. 2016 pp. 14-17 